\title{
El interferómetro de Michelson: entre el éter y las ondas gravitacionales*
}

\section{Michelson's Interferometer: Between Ether and Gravitational Waves}

Nalliely Hernández Cornejo ${ }^{\dagger}$

\begin{abstract}
Resumen
En este trabajo se presenta una reconstrucción de dos de los usos experimentales más sobresalientes del interferómetro de Michelson en la historia de la ciencia: la medición del éter $(1881,1887)$ y la detección de las ondas gravitaciones (2015). A partir de las diferentes interpretaciones de estos dos momentos estelares en la física, relativos a un mismo dispositivo experimental, me propongo recuperar algunas afirmaciones del filósofo Paul Feyerabend respecto a la relación entre teoría y experiencia, las cuales se ilustran plausiblemente con estos ejemplos. Al mismo tiempo, incorporo elementos sociológicos y psicológicos explorados por Harry Collins en sus estudios sobre ondas gravitaciones que pueden ser integrados plausiblemente en tal perspectiva, si bien esto tipo de elementos no suelen ser utilizados por el filósofo austriaco.
\end{abstract}

Palabras clave: interferómetro - éter - ondas gravitacionales - Feyerabend

\begin{abstract}
In this writing, I will briefly reconstruct the history of the Michelson-Morley experiment and the gravitational waves detection. Using these two conceptual frameworks for interpreting the same experiment in two stellar moments in history of Physics, I recover some Paul Feyerabend's statements concerning the relation between theory and experience. These statements, according to my view are plausible enlighten in this historical example. At the same time, I will include some sociological and psychological features about gravitational waves' detection that Harry Collins extensively examined, although these kind of elements are not regularly used by the Austrian philosopher.
\end{abstract}

Keywords: interferometer - ether - gravitational waves - Feyerabend

* Recibido: 22 de enero de 2018. Aceptado con revisiones: 7 de mayo de 2018.

† Universidad de Guadalajara, México. Para contactar al autor, por favor, escribir a: nallie3112@hotmail.com. Metatheoria 9(1)(2018): 29-49. ISSN 1853-2322. eISSN 1853-2330.

(C) Editorial de la Universidad Nacional de Tres de Febrero. Publicado en la República Argentina. 


\section{El éter y el experimento de Michelson y Morley}

Como es bien sabido, a finales del siglo XIX los físicos estaban convencidos de que las ondas electromagnéticas se propagaban en un material que llenaba todo el espacio, denominado éter. Fueron Thomas Young y Jean Fresnel los que reanimaron la teoría ondulatoria de la luz en los inicios de dicho siglo explicando los fenómenos de la difracción y la interferencial. ${ }^{1}$ Así, la teoría ondulatoria de la luz fue confirmándose y extendiéndose hasta que las nociones de la luz como corpúsculo, provenientes de la teoría newtoniana, se abandonaron por completo. ${ }^{2}$ Sin embargo, la analogía con el sonido, de la cual surgió el modelo ondulatorio de la luz, tendría que ser prontamente abandonada debido a que los fenómenos de polarización y doble refracción mostraron la propagación transversal (no longitudinal) de las ondas luminosas y, con ello, la analogía del calor radiante sustituyó la acústica (Swenson 1970, p. 57). Ahora bien, en este contexto sólo tenía sentido hablar de las ondas como perturbaciones que se propagan en un soporte material, respecto del cual se mide su velocidad, en el caso de las ondas electromagnéticas éste soporte era precisamente el éter. ${ }^{3}$

De esta forma, Michael Faraday, Heinrich Rudolf Hertz y James Clark Maxwell proporcionaron las leyes que describían los campos magnéticos y eléctricos concebidos como estados del éter. ${ }^{4}$

Con ello, la caracterización del éter como medio de propagación de las ondas electromagnéticas resultaba una creencia inherente y necesaria a la de la energía radiante para la década de 1880 (Swenson 1970, p. 57). Sin embargo, a diferencia de las ondas sonoras en las que su velocidad de propagación se mide utilizando el principio de relatividad, de acuerdo con el cual no hay un marco de referencia absoluto, sino que medimos el movimiento respecto de un punto que consideramos fijo, en el caso de las ondas electromagnéticas, el éter era identificado con el concepto de espacio absoluto de la física clásica, es decir, como el sistema de referencia fundamental ${ }^{5}$. Por otro lado, aunque los físicos estaban de acuerdo en que el éter debía ser muy ligero y difícil de comprimir para permitir que la luz viajara tan rápido como lo hace, el resto de sus características resultaron controvertidas. Por ejemplo, no estaba claro si se trataba de un éter inmóvil, sólo parcialmente arrastrado por la Tierra -como pensaba Fresnel${ }^{6}$ o si era completamente arrastrado por ella -como pensaba Stokes- ${ }^{7}$ en cuyo caso compartía su movimiento relativo en su superficie.

Eventualmente, la idea de Fresnel fue parcialmente adoptada debido a que pareció confirmarse por algunos experimentos como el de Fizeau de $1851 .{ }^{8}$ La idea de Fresnel implicaba que, si la velocidad del sonido es relativa a la del aire, la velocidad de la luz debía serlo respecto al éter. Por tanto, Tierra y éter están en movimiento relativo, lo cual suponía que un "viento del éter" debía existir y ser medible en

\footnotetext{
1 En 1678 (publicado en 1690) Huygens, en su Tratado sobre la luz, desarrolló una perspectiva ondulatoria de la luz análoga a la del sonido, "traduciendo" la óptica geométrica a una descripción ondulatoria y usando el éter como medio de propagación (Swenson 1972, pp. 9-10). En el siglo XIX la teoría de Fresnel unió la óptica de Huygens con el principio de interferencia de Young, dando por primera vez una explicación satisfactoria de la propagación rectilínea de la luz, así como de la existencia de franjas o bordes de difracción (Swenson 1972 , p. 20).

2 Hasta el surgimiento de la teoría cuántica a inicios del siglo XX, que recupera la noción corpuscular para describir a la luz.

3 En este contexto, una perturbación intuitivamente significaba perturbación de algo, es decir, de algún medio material.

4 El gran logro de Faraday y Maxwell fue sintetizar y unificar los fenómenos de la electricidad, el magnetismo y la óptica. Cuando sus predicciones se verificaron experimentalmente por parte de Hertz en 1887, los problemas de la física parecían dividirse en dos grandes categorías: los de la materia y los del éter (Swenson 1972, p. 28).

5 El electromagnetismo de Maxwell no era invariante respecto de las transformaciones de Galileo, por lo tanto, no cumplía con el principio de relatividad de la mecánica newtoniana, que en este contexto se traducía en la suma de velocidades entre marcos de referencia para pasar de uno a otro. De tal forma que, el éter llegó a considerarse el marco de referencia privilegiado respecto del cual se debía la velocidad de la luz.

${ }^{6}$ De acuerdo con Fresnel, el éter envuelto en medios ópticos comparte parte de su movimiento con éstos medios, dependiendo de sus índices de refracción. Los medios transparentes debían perturbar el éter de forma diferente en función del color de la luz, así la materia debía tener una distinta velocidad de perturbación para cada color (Einsenstaedt 2015, p. 204).

7 Retomando la hipótesis de Cauchy, Stokes defendía un éter totalmente perturbado y lo aborda desde un punto de vista hidrodinámico (Einsenstaedt 2015, p. 205).

8 Sin embargo, como el mismo Fizeau la afirma en su artículo de 1851, ninguna propuesta estaba libre de dificultades para establecer claramente las propiedades del éter y su relación con la materia (Einsenstaedt 2015, pp. 202-209). Durante la década de 1880 otros resultados negativos abogan a favor de la teoría de Stokes, que no obstante también podían ser explicados por la teoría de Fresnel.
} 
principio en la superficie terrestre. Ahora bien, en cualquier punto de la Tierra la magnitud y dirección del éter varía con el día y la estación, pero en algún momento debe coincidir con el movimiento terrestre, por lo que su análisis en diferentes momentos y direcciones debía posibilitar la determinación del movimiento relativo entre ambos. En síntesis, si era posible medir la velocidad de la luz (c) con suficiente precisión en estos diferentes momentos, entonces se podrían medir las velocidades de la luz a favor y en contra del viento etéreo respectivamente y compararse entre sí, como cuando se nada a favor y en contra de una corriente. ${ }^{9}$ La comparación entre las dos mediciones permitiría determinar la velocidad de éste. ${ }^{10}$

Albert Michelson, físico estadounidense de origen polaco, como oficial naval que era, pensó en una analogía que podría medir dicho viento: si la Tierra, moviéndose a través del sistema solar, era como un barco moviéndose a través del mar y del aire, entonces sería posible construir un medidor óptico suficientemente sensible de esa "corriente" para medir el movimiento relativo de la Tierra respecto del éter y convertir ese movimiento relativo en absoluto ${ }^{11}$ (Swenson 1970, p. 58). A partir de dicha analogía, Michelson diseñó un experimento para medir el efecto del viento etéreo creado por el movimiento de la Tierra a través de ese medio detectando patrones de interferencia creados por dos rayos de luz. Así nacería un exitoso dispositivo experimental denominado Interferómetro de Michelson, en sus palabras: "Durante el invierno de 1880 un método fue concebido para probar el movimiento relativo de la Tierra y el éter, el cual resultó en la invención del interferómetro". ${ }^{12}$

La idea de Michelson partía del supuesto de que la velocidad de la luz era constante y, dado que la Tierra se movía a través de un éter estacionario, el experimento estaba diseñado para detectar su orientación respecto a la propagación de la luz. De tal forma que cualquier diferencia en la propagación de la luz debía deberse a la disposición de la trayectoria de la luz en relación al movimiento de la Tierra a través del éter, es decir, asumía que la velocidad de la Tierra relativa al éter estacionario era la misma que la velocidad de la Tierra respecto al Sol (Staley 2008, p. 45). ${ }^{13}$

Michelson pensó en una forma de dividir un haz de luz en dos rayos en trayectorias perpendiculares, de tal forma que el instrumento sería orientado para que uno de los haces de luz viajara en dirección del movimiento de la Tierra en su órbita (este-oeste), mientras que el otro viajaría perpendicular a ésta (nortesur), y ello maximizaría la diferencia en velocidad respecto al viento etéreo creado por el movimiento de la Tierra (ver la figura 1). Asimismo, cambiar la orientación del instrumento indicaría cualquier cambio en la velocidad de la luz en los diferentes brazos del interferómetro, indicado en un cambio en el patrón de interferencia cuando la luz se recombinaba al llegar (Staley 2008, p. 48). Así, la expectativa fundamental era que la medición de un patrón de interferencia debía ser evidencia directa de una desviación del éter y, por tanto, de la velocidad de la Tierra respecto de éste (ver figuras 2 y 3 : la figura 2 describe la geometría del interferómetro y la figura 3 muestra un patrón de interferencia).

\footnotetext{
9 Aunque popularmente se suele explicar el experimento con la analogía de los dos nadadores en una carrera que lo largo y ancho de una corriente, Swenson ilustra las limitaciones de dicha analogía (1972, pp. 65-66).

${ }^{10}$ De acuerdo con la mecánica newtoniana la velocidad de la luz emitida por una fuente en movimiento (como la Tierra) debe diferir de su velocidad emitida por una fuente en reposo justamente una cantidad igual a la velocidad de la fuente $c^{\prime}=v^{+} c$. Entonces $c^{\prime}$ sería la velocidad de la luz relativa a la Tierra y $v$ la velocidad del éter relativa a la Tierra (Miller 1984, p. 104).

${ }^{11}$ Como afirma Swenson, la palabra 'absoluto' en este contexto se refiere a un marco de referencia mayor, aunque provisionalmente se pueda considerar el más grande y funcionar como el más fundamental de los sistemas inerciales de referencia (Swenson 1972, p. 41).

12 "During the Winter of 1880 a method was devised for testing the Relative Motion of the Earth and the Ether -which resulted in the invention of the Interferometer" (Michelson 1909, citado en Staley 2008, p. 28, traducción propia).

${ }^{13}$ Según Staley, la decisión de Michelson de dar este enfoque estuvo motivada por los comentarios de Maxwell, realizados en marzo de 1879 en una carta al director del Nautical Almanac, David Peck, en donde establecía que la velocidad de la Tierra respecto al éter altera la velocidad de la luz por una cantidad dependiente del cuadrado de la razón entre la velocidad de la Tierra y la de la luz (Ve/c) ${ }^{2}$. Al reducir estas expresiones obtenemos un efecto de segundo orden de 1/100000000, cantidad que describió como demasiado pequeña para ser observada (Staley 2008, pp. 45-46). Justamente, la particularidad del experimento de Michelson y la razón más importante para realizarlo es que fue concebido para ser capaz de medir un efecto de segundo orden (Swenson 1972, p. 66).
} 


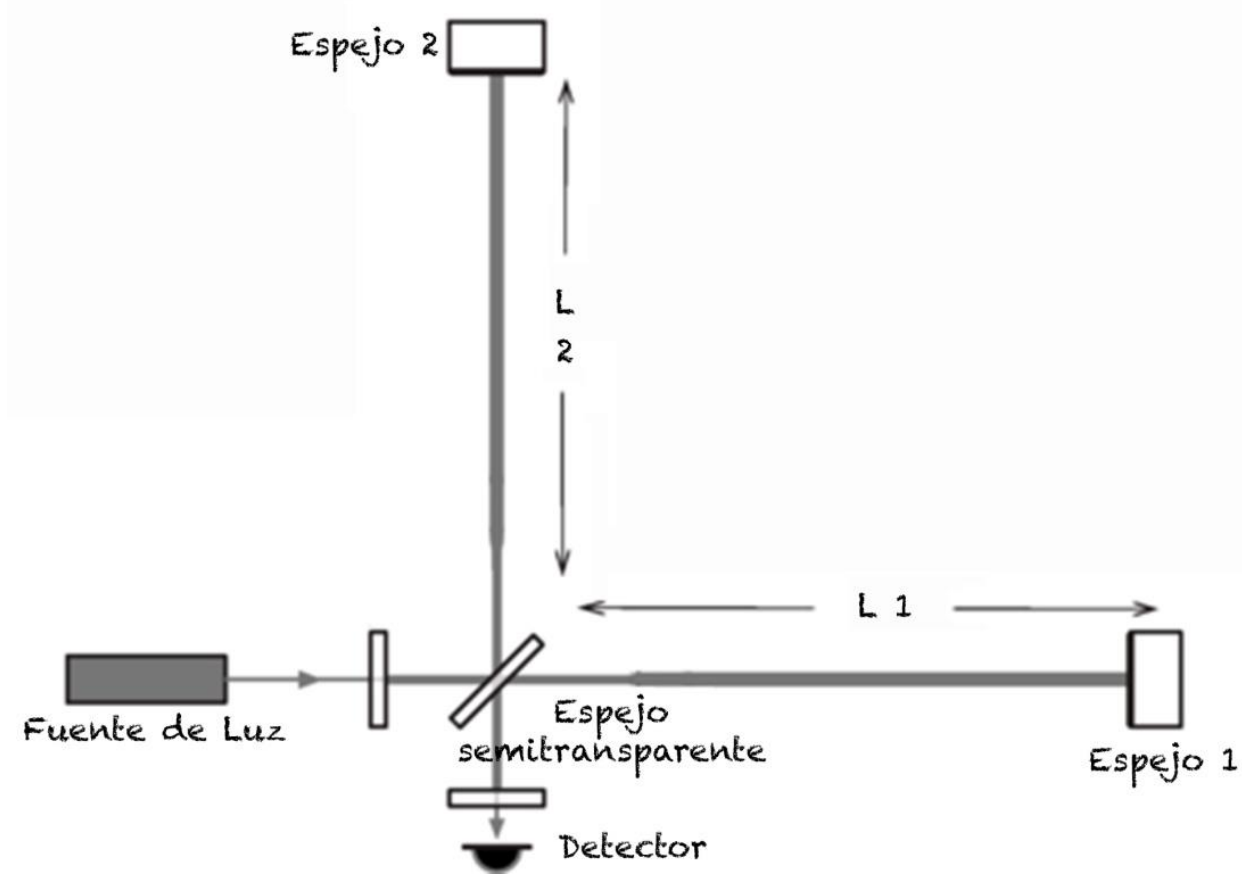

Figura 1. La fuente emite luz blanca y un espejo semitransparente los divide en dos haces. Estos viajan al final de los brazos 1 y 2 , donde re reflejan y vuelven a reunirse en el espejo inclinado (45 grados), donde hay un detector. Si la Tierra viaja a través del éter, un haz que se refleja paralelo al éter requerirá más tiempo en hacer el recorrido que el que lo recorre perpendicularmente- Debido al retraso se desfasan sus crestas y valles y esto causaría un patrón de interferencia, un desplazamiento entre áreas de la misma intensidad. El dispositivo se coloca en una mesa donde se rota para ver variaciones.
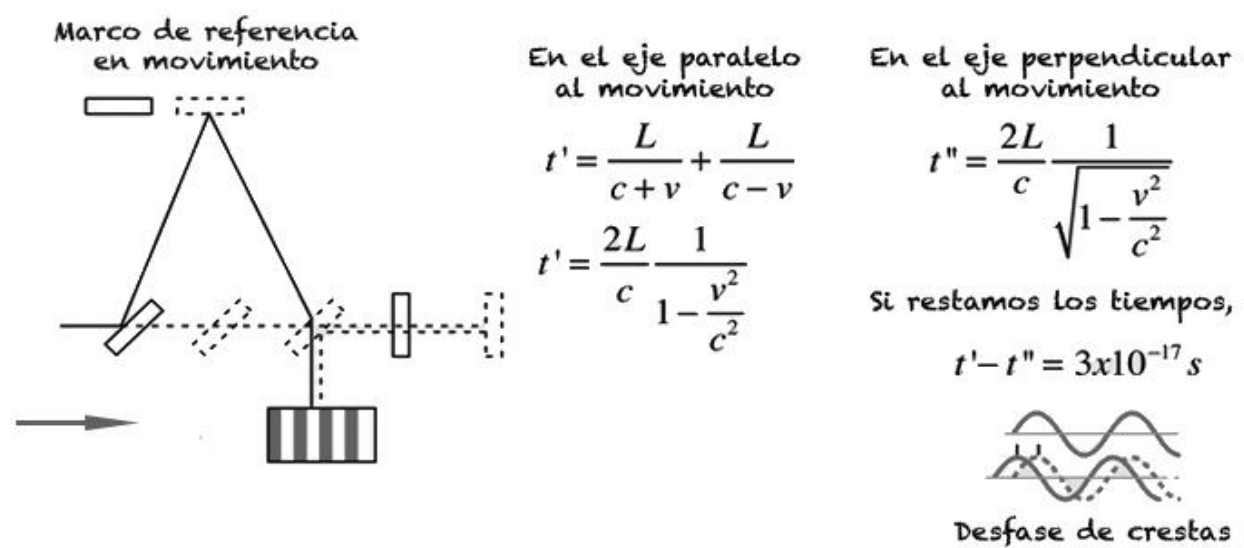

Figura 2. En un marco de referencia en movimiento en la dirección que indica la flecha tendríamos un rayo de luz que se mueve en el eje paralelo al movimiento, cuyo tiempo de recorrido para volver el primer espejo sería $t^{\prime}$ (haciendo algunas aproximaciones matemáticas), donde $\mathrm{v}$ es la velocidad del marco de referencia (la velocidad de la Tierra respecto al Sol) y c es la velocidad de la luz. En el caso del rayo perpendicular al movimiento del marco de referencia, para que llegue a golpear el espejo ha recorrido una distancia $\mathrm{ct}^{1}$. Pero el espejo ha viajado una distancia $=v \mathrm{t}^{1}$ en la dirección $\mathrm{x}$ (la dirección de $\mathrm{v}$ ). Usando el Teorema de Pitágoras la distancia que recorre este rayo de luz es $\sqrt{L^{2}+\left(v t^{1}\right)^{2}}=\mathrm{ct}^{1}$. Por lo tanto, el tiempo de ida del rayo es $\mathrm{t}^{1}=\mathrm{L} / \sqrt{L^{2}+\left(v t^{1}\right)^{2}}$, que es el mismo tiempo $\mathrm{t}^{2}$ que tarda en volver. Sumando los tiempos, $\mathrm{t}^{\prime \prime}=\frac{2 L}{\sqrt{c^{2}-v^{2}}}$ que usando algunas aproximaciones matemáticas resulta en la expresión de la figura. Si restamos los tiempos y sustituimos los valores de v y c podemos obtener una aproximación del 
desfase de las ondas y con ello calcular el desfase de las crestas, por tanto, el patrón de interferencia generado.

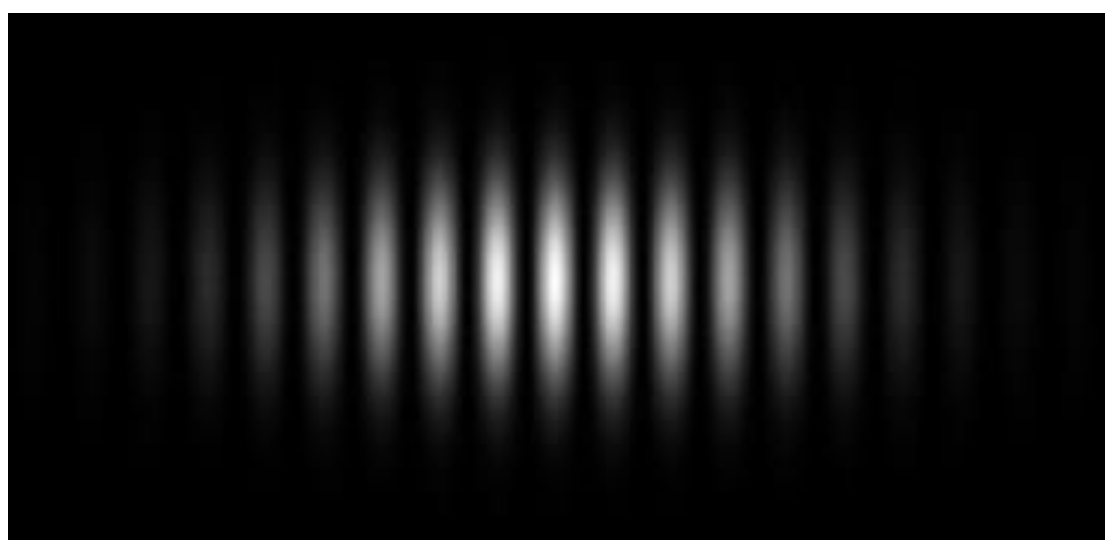

Figura 3. Patrón de interferencia esperado de luz monocromática coherente

Michelson realizó el experimento en 1881 y publicó un artículo donde describe su hipótesis, el aparato y una tabla de resultados que mostraban 4 diferentes series de observaciones con el interferómetro girado 90 grados entre cada serie. El resultado reporta que no obtuvo un desplazamiento significativo de las bandas de interferencia (los desplazamientos obtenidos eran atribuibles a errores de experimentación) (Swenson 1970, p. 59). La conclusión, en palabras del propio Michelson fue: "El resultado de la hipótesis de un éter estacionario es, por tanto, incorrecta" (Michelson 1881, p. 122). Sin embargo, los físicos Alfred Potier y Henri A. Lorentz mostraron que sus cálculos eran erróneos al haber despreciado un pequeño efecto del éter en el movimiento del rayo perpendicular a la rotación de la Tierra (Staley 2008, p. 55). Además, éste último pensaba que la longitud de los brazos era muy pequeña para una medición definitiva de segundo orden.

En 1886 Michelson, junto con Edward Morley, comienza a rediseñar el experimento, incrementando la longitud de la trayectoria de los rayos y reduciendo las probabilidades de vibración, las dos deficiencias más importantes del aparato de 1881. Utilizaron una serie de espejos para reflejar la luz repetidamente para incrementar considerablemente el efecto, y Morley sugirió hacer flotar el dispositivo en mercurio para resolver el problema de la vibración (Staley 2008, p. 60). Así, en Julio de 1887, Michelson y Morley realizaron un nuevo conjunto de observaciones que consistían en 36 giros del interferómetro que a su vez cubrían un total de 6 horas de duración repartidas en un periodo de 5 días. Esperaban encontrar un desplazamiento de bandas de 0.4 , pero reporte mayor fue $1 / 20$ de su predicción de la distancia desplazada entre franjas (Swenson 1970, p. 59). Su conclusión fue que: "Si había algún desplazamiento debido al movimiento relativo entre la Tierra y el éter luminífero, ésta no podía ser mucho mayor que 0.01 de la distancia entre las franjas" (citado por Staley 2008, p. 60). La velocidad medida fue considerada demasiado pequeña para ser usada como evidencia de la velocidad relativa al éter, y se consideró dentro del rango de error experimental. Con ello se concluía que la Tierra se mueve a través del éter sin ningún tiempo de arrastre o fricción, pues éste no es afectado por la materia que permea (Swenson 1972, p. 87).

Aunque este resultado rompía con parte de la teoría del momento, podía ser considerado como no concluyente debido a que habían tomado en cuenta sólo el movimiento orbital de la Tierra $(30 \mathrm{~km} / \mathrm{s})$, habiendo despreciado el (desconocido) movimiento del sistema solar, que podría -aunque lo consideraban improbable- modificar ligeramente el resultado. Además, una segunda limitación surgía si se admitía la posibilidad de que en un ambiente menos protegido que un sótano (arriba del nivel del mar) como una montaña, el movimiento del éter pudiera ser más perceptible (Staley 2008, pp. 60-61). De forma extraña, aunque ambas limitaciones sugerían replicar los resultados y ellos mismos afirmaron que la medición debía repetirse en intervalos de tres meses para evitar la posibilidad de tal incertidumbre, ello ya no ocurrió (Staley 2008, p. 62). 


\section{Las interpretaciones del experimento: Lorentz, Einstein y la existencia del éter}

La historia de la explicación del experimento de Michelson y Morley (M\&M), a pesar de su fama, es compleja e involucra varias etapas y perspectivas. Si bien en el ámbito especialmente pedagógico de la física suele tomarse como el famoso experimento crucial que refutó la existencia del éter, dando pie al origen de la relatividad, ésta es una lectura a posteriori y simplificada que ha sido ampliamente objetada por la historia y la filosofía de la física. ${ }^{14}$ De hecho, las reacciones inmediatas del experimento de 1887 fueron prácticamente despreciables, con la excepción de George Francis FitzGerald, como veremos más adelante.

Como se ve en las conclusiones de los autores del experimento, lo que ellos afirman es que la hipótesis del éter estacionario que produce arrastre en el movimiento de la Tierra es falsa, pero no concluyen su inexistencia. Efectivamente, Michelson inicialmente pensó que su experimento llevaría a la confirmación de la teoría de Stokes, de acuerdo con la cual el éter era completamente arrastrado en la vecindad de la Tierra. De tal forma que otras versiones del experimento después de 1887 se realizaron con mayor sofisticación (ver Swenson 1970). Muchos experimentalistas continuaron intentando medir diferencias relativas de movimiento, suponiendo que el experimento del interferómetro no había sido suficientemente preciso. ${ }^{15}$ Científicos como Olvier Lodge, Jospeh Larmor, Arthur Schuster Gustave LeBon o Joseph J. Thompson fueron algunos de los más importantes defensores del éter durante la década de 1910 (Swenson 1970, p. 61).

Por otro lado, en el ámbito teórico Lord Kelvin, Henri Poincaré y Henri A. Lorentz, entre otros, teorizaron sobre las anomalías que suponía el experimento y sus posibles soluciones. Un primer paso para explicar los experimentos de M\&M fue la hipótesis de la contracción de Fitzgerald-Lorentz (LFC), presentada primero por George Fitzgerald ${ }^{16}$ en 1889 y luego por Henri Lorentz en 1892. De acuerdo con ella, los objetos se contraen en la dirección del movimiento, originalmente pensado en relación al éter. De hecho, si la contracción de L se inserta en el cálculo del experimento, los tiempos de recorrido en los brazos se igualan y ello explica la falta del patrón de interferencia. ${ }^{17}$

Sin embargo, la explicación que Lorentz propone ya para 1904 se inserta en un cuadro teórico más completo. El físico neerlandés partía del supuesto de la existencia del éter y de unas partículas pequeñas que poseen masa y carga eléctrica (electrones). Estos electrones en su movimiento a través del éter generan un campo electromagnético, de tal forma que usando las ecuaciones de Maxwell para un campo fijo en el éter se debería poder determinar el campo magnético a partir de la carga, posición y estado de movimiento de los electrones, es decir, de la distribución de carga y velocidades. Ello llevó a Lorentz a una ecuación diferencial cuya solución es válida sólo en un sistema de coordenadas que está en reposo. Sin embargo, la Tierra se movía a través del éter y debido a que nuestras mediciones son relativas a este

\footnotetext{
${ }^{14}$ Normalmente en la pedagogía de la física se hace esta lectura porque se considera que la existencia del éter (p) implicaba que el resultado del experimento tuviera un resultado positivo $(\mathrm{p} \rightarrow \mathrm{q})$, por lo que el resultado negativo implicaba la negación de la hipótesis del éter $(\neg \mathrm{q}$ $\rightarrow \neg$ p). Sin embargo, por un lado, muchos científicos no consideraron concluyente el resultado y, por otro, habría otras caracterizaciones del éter que no implicaban necesariamente un resultado positivo del experimento de $\mathrm{M} \& \mathrm{M}$ (por lo que no necesariamente $\mathrm{p} \rightarrow \mathrm{q}$ ). En todo caso, el experimento solo refutaba la versión del éter estacionario.

${ }^{15}$ Miller trabajó en interferómetros cada vez más grandes, con brazos hasta de $32 \mathrm{~m}$ de largo, en una montaña y de mucho ruido obtuvo un pequeño resultado variable. Estos resultados fueron discutirlos por Michelson y Lorentz muchos años después, pero no eran concluyentes. Se intentaron modificaciones técnicas para aumentar la precisión, pero no se llegó a ninguna conclusión definitiva.

${ }^{16}$ FitzGerald propuso que el resultado del experimento podía ser explicado suponiendo que el tamaño de los objetos es una función de su velocidad a través de éter. De esta forma, los cuerpos cambian sus dimensiones dependiendo de su movimiento absoluto respecto del éter, por lo que los brazos del interferómetro eran deformados lo suficiente para compensar exactamente el desplazamiento esperado, debido a una distorsión electromagnética en su movimiento a través del espacio. Lorentz algunos meses más tarde e independientemente de FitzGerald propondría una solución similar (Swenson 1972, p. 108).

${ }^{17}$ De acuerdo con LFC un cuerpo a través del éter con velocidad $v$ se acorta por un factor $\sqrt[2]{1-\frac{\mathrm{v}^{2}}{\mathrm{c}^{2}}} \cdot \mathrm{r}$.
} 
marco de referencia, el físico consideró unas ecuaciones de campo en un marco de referencia sujeto a un objeto en movimiento de donde las ecuaciones obtenidas resultaban más complicadas que las de Maxwell. De tal forma que introdujo unas transformaciones como herramienta matemática para que la solución de una de las ecuaciones llevara a la solución de la otra (Zahar 1973a, p. 112). Así, aplicando estas transformaciones espacio-temporales de Lorentz obtenemos los estados correspondientes en distintos sistemas de referencia inerciales, de tal forma que las ecuaciones de Maxwell tienen la misma configuración en cualquier marco de este tipo que se mueve en el éter (Miller 1984, p. 105). Pero, según Lorentz, estos representan campos ficticios, en términos de coordenadas espacio-temporales ficticias que podemos traducir a su configuración real. Como resultado, interpreta esta transformación de coordenadas en el caso de la longitud como la contracción (LFC) que explica el resultado del experimento. ${ }^{18}$

De acuerdo con la lectura más o menos canónica y popular del folklore y mito científico, la contracción LFC es una hipótesis auxiliar que resulta ad-hoc, característica que Einstein identificó como insatisfactoria y que le motivó para elaborar la Teoría Especial de la Relatividad (Janssen 2002, p. 432). Por ejemplo, para Popper la LFC resulta ad-hoc porque que no tiene consecuencias falsables, sino que sólo sirve para restituir el acuerdo entre teoría y experimento en el caso del experimento de M\&M (citado por Zahar 1973a, p. 99). De forma similar, para Holton (1969) la hipótesis resulta ad-hoc porque no está integrada en el resto de la teoría de Lorentz y porque fue elaborada específicamente para dar cuenta de éste experimento (Zahar 1973a, p. 99). Sin embargo, ambas afirmaciones parecen estar equivocadas.

Aunque Holton (1969) argumenta que el experimento no tuvo un papel central en las consideraciones de Einstein en torno a la relatividad, ${ }^{19}$ según él, el carácter ad-hoc de la explicación de Lorentz sería un signo de la superioridad de la solución de Einstein. No obstante, de acuerdo con Zahar (1973a), la hipótesis de la contracción no puede ser ad-hoc en el sentido de Popper porque ésta puede ser confirmada por experimentos diferentes al de $M \& M$, por lo que puede ser probada independientemente de éste. ${ }^{20}$ De igual forma, como antes hemos descrito, Zahar afirma que Lorentz deduce la LFC de una teoría más general en la que las fuerzas moleculares se comportan y transforman como fuerzas electromagnéticas. De tal forma que, la hipótesis de las fuerzas moleculares es estructurada de acuerdo con la heurística del modelo del éter, que requiere que los fenómenos se expliquen en términos de la propagación del éste y su interacción con la materia, y en la deducción de la contracción usa las transformaciones en tanto que propiedades de las ecuaciones de Maxwell (Zahar 1973a, pp. 105-106). Ello parece mostrar que la hipótesis está integrada en la teoría de Lorentz y su desarrollo es independiente del experimento, si bien está claro que una de las preocupaciones de Lorentz al desarrollarla es explicar el resultado del mismo sin contradecir las leyes de Newton y las de Maxwell. ${ }^{21}$

Por lo tanto, al menos en la versión madura de lo que Lorentz llamará el Teorema de los Estados Correspondientes, resulta natural agregar la hipótesis de la contracción generalizada y esto permite derivar la idea de que el movimiento de la Tierra a través del éter no será detectable en el experimento. ${ }^{22}$ Lo que significa que la hipótesis no resulta ad-hoc, cuestión relevante para afirmar la consistencia y racionalidad de la propuesta de Lorentz. Esto nos lleva a la tercera interpretación del experimento.

\footnotetext{
${ }^{18}$ En las transformadas también hay una "coordenada del tiempo local", pero el tiempo real seguía siendo el tiempo newtoniano (Miller 1984, p. 105).

${ }^{19}$ Sin embargo, no hay consenso al respecto, pues el propio Einstein parece darle diferente peso en distintos momentos de su vida (ver Swenson 1972, p. 158).

${ }^{20}$ Por ejemplo, el experimento tipo Kennedy-Thorndike descrito por Grünbaum (ver Zahar 1973).

${ }^{21}$ Sin embargo, Janssen niega la argumentación de Zahar citando una carta en la que Lorentz admite que encontró el argumento de las fuerzas moleculares para elaborar la hipótesis de la contracción sólo después de notar que dicha hipótesis explicaba el resultado del experimento. Además, afirma, confunde plausibilidad con derivación concluyendo que no hay tal conexión entre el tratamiento electrostático y el óptico basado en el teorema de estados correspondientes en la teoría temprana de Lorentz, solamente en su versión madura aparece la conexión aludida por Zahar (ver Janssen 2002, pp. 436-437).

${ }^{22} \mathrm{Si}$ un observador en la Tierra, en el marco de referencia que se mueve en el éter, siempre mide las cantidades o transformadas ficticias para ese marco, que satisfacen las mismas ecuaciones que las cantidades reales en un marco de referencia en el éter, se sigue que el observador nunca podrá detectar el movimiento del éter.
} 
Albert Einstein, al publicar su famoso artículo sobre la Teoría Especial de la Relatividad (1905) -a diferencia de Lorentz y su teoría dinámica sobre el electrón-, introduce un marco cinemático general que consiste en principio universal de covarianza (el principio de relatividad) y el postulado sobre la constancia de la velocidad de la luz. A partir de este postulado el experimento queda automáticamente explicado o, mejor dicho, el resultado no requiere explicación puesto que coincide con él, pues la velocidad de la luz no sufre variaciones. Este postulado, de acuerdo con algunos autores (Kompaneyets 1962) constituye una generalización del resultado de $M \& M$ y, por tanto, supera la explicación de Lorentz. Sin embargo, para otros como Reichenbach, no constituye una explicación del experimento, ya que el resultado es simplemente aceptado como un axioma (Reichenbach 1958, p. 201), del cual el resultado de $M \& M$ se sigue trivialmente y que además no resulta en absoluto intuitivo (Zahar 1973a, p. 108). Más aún, según el mismo Zahar, la teoría de Lorentz no solamente explica el resultado del experimento, sino la propia invarianza de la velocidad de la luz, ya que ésta es deducible de su teoría, mientras que en Einstein permanece como un postulado. De esto se sigue que la superioridad que mostrará eventualmente la teoría de Einstein debe residir en otras razones, pero no en el carácter ad-hoc de la contracción o una explicación más satisfactoria del experimento de M\&M (Zahar 1973a, pp. 108-109).

Ahora bien, en el artículo de Einstein las transformaciones de Lorentz son usadas para relacionar las coordenadas de dos marcos de referencia inerciales, pero dando una interpretación realista del sistema en movimiento, y con ello, las coordenadas obtenidas son reales, no ficticias, y las transformaciones de Galileo son definitivamente eliminadas. ${ }^{23}$ En definitiva, para el físico alemán, las cantidades provenientes de las transformadas de Lorentz son las cantidades reales correctamente medidas. De tal forma que, como Poincaré demostró, ambas teorías son empíricamente equivalentes, pues las cantidades "efectivas" o medidas son las mismas, aunque tienen diferentes interpretaciones. Tan es así que en 1905 no se concebían como propuestas claramente diferenciadas y a menudo se les refería como la teoría LorentzEinstein (Staley 2008, p. 254).

Así, podemos contrastar ambas propuestas teniendo en cuenta que, en la teoría dinámica de Lorentz, tiempo y espacio absolutos, por tanto, el concepto de éter, tienen un papel fundamental, pues el hecho de que la medición de la velocidad de la luz sea siempre la misma es resultado de la interacción de los electrones con éste (Miller 1984, p. 108). Estos son los elementos que permiten conservar un mundo físico clásico que reconstruyera y armonizara la mecánica newtoniana y el electromagnetismo de Maxwell (Zahar 1973a, p. 122). Como consecuencia, en la versión final de su teoría las medidas nunca revelan las cantidades reales porque todas están hechas con instrumentos que están distorsionados e inadecuadamente sincronizados como resultado de su movimiento y el del observador respecto del éter, es decir, de ese espacio y tiempo absolutos (Janssen 2002, p. 429). Por otro lado, para Einstein la ciencia debe proporcionar una descripción de la realidad coherente, unificada, armoniosa, simétrica ${ }^{24}$ y simple a partir de un mínimo de conceptos y relaciones básicos (Zahar 1973b, p. 224). Sin embargo, las asimetrías que se presentaban entre la teoría newtoniana y electromagnética, entre partículas y campos, ${ }^{25}$ o peor aún, la incompatibilidad que surgía de aplicar el principio de relatividad y con él las transformaciones de Galileo a la primera, ${ }^{26}$ pero no a la segunda, impedían formular una unidad de fundamentos físicos de los que Einstein estaba convencido.

\footnotetext{
${ }^{23}$ De hecho, las propiedades matemáticas de las transformaciones aseguran que la situación de un observador en reposo en el éter visto desde el punto de vista de un observador en movimiento a través del éter es la misma situación de un observador visto desde el punto de vista del observador en reposo. De tal forma que no hay forma de decir cuál de los dos observadores está verdaderamente en reposo respecto del éter (Janssen 2002, p. 428).

${ }^{24}$ Es bien conocido que Einstein estaba ocupado en el problema de que la teoría electromagnética diera dos explicaciones diferentes para el problema de la inducción entre un conducto o un imán, dependiendo cuál de ellos estuviera en movimiento, aunque su efecto físico medible dependiera de la velocidad relativa entre ellos (Miller 1981, pp. 114-115). Por lo tanto, era una prioridad establecer la equivalencia entre los puntos de vista ente observadores en movimiento (Millar 1981, p. 117).

${ }^{25}$ Einstein señala que la noción newtoniana de materia puntual y la de campo como un continuo, como conceptos elementales, hacen que la energía cinética y de campo respectivamente aparezcan como cosas diferentes (Zahar 1973b, p. 238).

${ }^{26}$ Estas permiten pasar de un marco de referencia a otro en la mecánica clásica.
} 
De esta forma, la decisión de Einstein fue extender el principio de relatividad a la electrodinámica usando las transformaciones de Lorentz para eliminar la asimetría y, por tanto, modificar la cinemática clásica y con ello, la idea de movimiento absoluto (Zahar 1973b, p. 230). En definitiva, la asimetría introducida por la diferencia entre un sistema asociado al éter y el resto de sistemas de referencia llevó al físico a desafiar la propia existencia del éter (Kostro 2000, p. 19). Así, como el propio Einstein afirma en su artículo, las mismas leyes serían válidas para la óptica y la electrodinámica en todos los marcos de referencia inerciales (Einstein 1923, p. 38). Asimismo, su referencia a los fracasos para detectar el movimiento de la Tierra a través del éter alude indirectamente al experimento de $M \& M$ que parece ser usado como soporte del segundo postulado -la invarianza de la velocidad de la luz-, como afirman Holton (1969, pp. 164-165) y Zahar (1973b, p. 232). Sin embargo, como éste último apunta, el postulado parece salir de dar por sentada la corrección de las ecuaciones de Maxwell (como teoría unificada y confirmada) con las cuales el segundo postulado es consistente (Zahar 1973b, p. 233). De esta forma, desde el punto de vista lógico, a partir de admitir los dos postulados que son supuestos más débiles se puede desarrollar una nueva cinemática y derivar las transformaciones de Lorentz. Dichas transformaciones se convierten así en una condición general de cualquier teoría física, trascendiendo su relación con la teoría electromagnética y, sobre todo, reflejan una nueva naturaleza y estructura del espacio y el tiempo (Zahar 1973b, p. 235). Así, espacio y tiempo absolutos resultan rechazables por su carácter metafísico. Esta actitud, mantenida por Einstein, a menudo se señala como producto de la influencia, según diversas fuentes, de su lectura de David Hume, Poincaré y Ernst Mach (Miller 1984, p. 116).

Por tanto, podemos ver que al menos tenemos tres grandes lecturas de la ausencia del patrón de interferencia en el dispositivo de M\&M: a) la de aquellos experimentalistas que insistieron en detectar el éter, al considerar no concluyente el resultado; b) la de Lorentz y sus seguidores, que intentaron explicar la ausencia del patrón a partir de la existencia del éter y las teorías clásicas de la mecánica y el electromagnetismo; y c) la einsteiniana, en la que el resultado era una consecuencia inmediata de la invarianza de la velocidad de la luz y que partía de una nueva cinemática y, por tanto, de una nueva estructura del espacio y del tiempo, donde el éter era prescindible.

La primera respuesta se fue debilitando paulatinamente, ante la ausencia de resultados significativos en interferómetros y a través de otras técnicas La elección entre la segunda y la tercera respuesta es más complicada. Como hemos dicho, ambas teorías eran empírica u observacionalmente equivalentes. Por ello la revolución einsteiniana se hizo evidente cuando los científicos tomaron parte a partir de las propuestas disponibles (aunque no todos lo hicieron), sin ningún experimento que ofreciera pruebas definitivas e incontrovertibles de su aproximación. ${ }^{27}$ En lugar de estas, una lenta erosión de las distintas conceptualizaciones del éter fue ocurriendo, lo que permitió ganar terreno a la propuesta relativista. No hay consenso sobre exactamente cuándo y cómo ocurrió esto. Para algunos (Zahar, por ejemplo) fue su poder heurístico el que generó que se mostrara superior, al menos hasta el surgimiento y las primeras comprobaciones de la teoría de la relatividad general, cuando se mostró empíricamente superior. Sin embargo, esta posibilidad parece difícil durante 1906 a 1908, ya que pocos habían entendido realmente la propuesta relativista para este momento. Para otros su éxito radica los principios "simples", generales, simétricos y económicos que subyacen en la descripción einsteiniana (Janssen 2002).

Por otro lado, para algunos la disputa estaba fundamentalmente dirimida en 1911, aunque varios se subieron al carro einsteiniano antes (Planck), mientras que para otros tardó más tiempo. De hecho, algunos estudios afirman que existieron diferentes respuestas ante la relatividad en función de las diferentes tradiciones científicas en distintos lugares. Mientras que en Alemania se discutió y estudió seriamente, no fue así en otros lugares como Francia, Gran Bretaña o Estados unidos (Goldberg 1970). De acuerdo con Swenson (1972), para 1910 el escenario estaba puesto para un duelo final entre aquellos que pensaban que no podría haber ondas a menos que fueran ondas en "algo" y aquellos que, junto con

\footnotetext{
${ }^{27}$ Por ejemplo, los experimentos de Bucherer eran consistentes tanto con la teoría de Lorentz como la de Einstein, los de Kaufmann refutaban ambas (Zahar 1973b, p. 259). Más tarde resultó que ambos resultados eran incorrectos.
} 
Einstein, pensaban que el éter era superfluo, ${ }^{28} \sin$ embargo, la confusión entre los argumentos a favor y en contra de la relatividad era grande y la delgada línea entre afirmaciones físicas y metafísicas era traspasada frecuentemente por uno y otro lado (Swenson 1972, p. 183).

En cualquier caso, la decisión por una u otra hasta antes de 1916 estaba dada por una carga teórica de principios físicos, preferencias epistémicas e idiosincrasias personales, que podían o no estar insertadas en tradiciones locales, pues como hemos dicho ambos planteamientos son racionales y empíricamente equivalentes. Adicionalmente, mientras que Lorentz es un físico famoso y reconocido, Einstein es relativamente desconocido, lo cual dificulta explicar la aceptación de su propuesta, cuyo precio implica revisar las nociones de tiempo y espacio de una forma que para entonces resultaba muy poco intuitiva. Por lo tanto, la cuestión parece dirimirse entre aquellos que les parecía más plausible conservar las teorías físicas clásicas y con ellas, la enraizada noción del éter al precio de complejizar la explicación, y aquellos que optaban por un par de postulados económicos, pero nada intuitivos y una estructura teórica más sencilla y simétrica al precio de reformular la estructura espacio-temporal -y con ello la mecánica entera. Así, la nueva teoría parecía deshacerse de una metafísica redundante que, según algunos, albergaba la teoría de Lorentz (Bridgman 1936, Eddington 1939).

Sin embargo, la historia del éter no terminaría ahí, pues el concepto del éter fue retomado por el propio Einstein años más tarde en el marco de su Teoría de la Relatividad General (1916) y por otros físicos más adelante -como Dirac o Weyl (Kostro 2000)-. Si bien, está claro que estas nuevas elaboraciones no corresponden en absoluto a la noción defendida y caracterizada por Maxwell, Lorentz o Poincaré en tanto que marco de referencia absoluto y medio en el que se propagan las ondas electromagnéticas. Einstein se referirá más bien a un éter mucho más abstracto y constitutivo geometrodinámico del espacio-tiempo que determina la interacción gravitacional de la materia (Kostro 2000, pp. iii). Este éter ahora expresaba las propiedades físicas y geométricas del espacio. Pero esa es ya otra historia.

\section{La existencia de las ondas gravitacionales y el interferómetro de Michelson}

Cómo hemos visto ya, en 1905 -con la elaboración de la Teoría Especial de la Relatividad por parte de Einstein- se inauguraban unos nuevos fundamentos cinemáticos que implicaban a su vez las modificaciones de las nociones clásicas de espacio y tiempo. Posteriormente, entre 1915 y 1916, Einstein generaliza su teoría en la Teoría General de la Relatividad, donde propone una explicación de la gravitación a partir del concepto de curvatura del espacio-tiempo o geometrización de la gravitación. Se trata de una teoría de campo de la gravitación que usa hasta cierto punto el electromagnetismo como guía. Sin embargo, la gravedad no es un campo extra sobre el espacio, sino que la gravedad una manifestación geométrica del espacio-tiempo.

En la teoría de la Relatividad General se puede pensar la estructura del espacio-tiempo como un tejido flexible que puede deformarse. De tal forma que, entre sus predicciones se encuentra la existencia de ondulaciones o perturbaciones del espacio-tiempo producidas por cuerpos masivos acelerados denominados ondas gravitacionales, que viajan a la velocidad de la luz. Originalmente fue el mismo Einstein quien propuso la existencia de las ondas gravitacionales y sus propiedades en 1916 (Abbott 2016, p. 1). Sin embargo, no se tenía claridad sobre si se trataba de un efecto físico o matemático de la teoría. En un artículo de1937 el físico cree demostrar en un caso particular que éstas no existen (Einstein \& Rosen 1937); sin embargo, más tarde quedó claro que este resultado estaba equivocado. Para la década de 1960 había un amplio consenso sobre que las ondas eran emitidas y que eran en principio detectables, es decir, que tenían un efecto sensible por las cosas a través de las cuales pasaban (Collins 2004, p. 67).

\footnotetext{
${ }^{28}$ También hubo un conjunto de intentos para romper la diferencia entre relatividad y éter. Por ejemplo, en 1914 Leigh Page intentó caracterizar un éter consistente con el principio de relatividad (Swenson 1972, p. 182).
} 
En concreto, la teoría de la relatividad nos dice que, bajo circunstancias extremas, cuando las cosas se aproximan a la velocidad de la luz y colisionan, la masa comienza a incrementar mientras que el tiempo se dilata y se vuelve muy lento. Análogamente a las ondas electromagnéticas que se producen por cargas aceleradas, las ondas gravitacionales se producen cuando se aceleran masas o se ponen a oscilar y pierden energía hasta colapsar. Mientras ocurre esto, en la vecindad de estos objetos el espacio-tiempo es "sacudido", y esta sacudida espacio-temporal constituye una onda gravitacional en la que las vibraciones se propagan a través del universo como ondas en un estanque en el que las distorsiones son transversales a la dirección de propagación, ${ }^{29}$ Sin embargo, debido a la rigidez del espacio, las ondas son mínimas y se van debilitando conforme se propagan en todas las direcciones y en las enormes distancias del espacio. Por tanto, resultan muy difíciles de detectar y se requiere un instrumento extraordinariamente sensible para hacerlo (Collins 2004, p. 4).

Ahora bien, se requiere que un evento violento y dinámico sea gravitacionalmente asimétrico para que las ondas sean emitidas. De acuerdo con ello, la fuente potencial de esta energía de las ondas gravitacionales debía ser la interacción de dos objetos masivos, el decaimiento de la órbita de cuerpos o la reducción de sus masas (Collins 2004, p. 69). En la misma década de 1960, científicos como Richard Feynman, John Wheeler, Herman Bondi o Felix Pirani concluyeron que las ondas gravitaciones eran emitidas por sistemas que giraban uno alrededor del otro y se propusieron calcular sus efectos (Collins 2004, p. 73). Por ejemplo, si en un sistema binario de estrellas de neutrones una oscila alrededor de la otra, al final de su vida la espiral final produciría una chirp de radiación gravitacional que deformaría el espacio-tiempo y, por tanto, los objetos en él (Collins 2004, p. 71).

De acuerdo con lo anterior, para ver ondas gravitacionales tendríamos que ser capaces de ver efectos como éste en las distancias entre las cosas. Es decir, tendríamos que medir el cambio de distancias entre dos objetos cuando la onda gravitacional pasa, pero esta distancia es tan pequeña que es equivalente a ver el grosor de un cabello humano a la distancia entre la Tierra y la estrella más cercana (Collins 2004, p. 3).

Los físicos debatieron largamente sobre la posibilidad real y práctica de detectar estos fenómenos, pues durante varios años sólo se tenían algunas mediciones indirectas de su existencia (Taylor \& Weisberg 1982). Fue Joe Weber quien abrió de forma definitiva dicha posibilidad gracias proponiendo y llevando a cabo la construcción de un posible detector (Taylor \& Weisberg 1982, p. 30). Estos detectores consistían en grandes cilindros o barras de metal encerrados al vacío que debían vibrar en respuesta al paso de una onda gravitacional, ${ }^{30}$ Como resultado de este trabajo, Weber anunció la detección de las ondas gravitacionales a finales de la década de los 60s, sin embargo, su trabajo nunca pudo ser replicado ni justificado teóricamente. ${ }^{31}$ Por lo tanto, con el tiempo dicho resultado terminó por ser rechazado por la comunidad científica. Paradójicamente, otras publicaciones controvertidas al respecto de un grupo en Roma que usaba barras criogénicas, junto con el caso Weber, abrieron e impulsaron este campo, pero al mismo tiempo sembraron muchísimas reservas sobre los estándares admitidos y los cuidados que una detección exitosa debía implicar (Collins 2004).

Por otro lado, Michelson había iniciado y desarrollado un programa técnico que contribuyó a una nueva cultura de la instrumentación alrededor de las técnicas de la interferometría a principios del siglo XX (Staley 2008). Debido a la geometría del interferómetro, que se explicó brevemente en el contexto del éter, éste resulta un instrumento ideal para medir distancias y cambios de distancia muy pequeños.

\footnotetext{
${ }^{29}$ Los componentes de la métrica que describen el espacio-tiempo con una ecuación de onda dada por la ecuación diferencial: $\frac{1}{c^{2}} \frac{\partial^{2} h}{\partial t^{2}}=$ $\nabla^{2} h$. La solución de esta ecuación (h) es una onda que indica que el vació del espacio “se estira” temporalmente en una dirección, mientras se contrae en otra.

${ }^{30}$ Hubo una primera generación de estos detectores durante los años 60 s y 70 s del siglo pasado y una segunda generación de detectores que estaban congelados a la temperatura de Helio líquido por lo que se conocieron como barras criogénicas. Las barras de aluminio tienen frecuencias naturales de oscilación iguales a las de los sistemas físicos que se quieren medir por lo que la onda hace que el sistema entre en resonancia y vibre con la misma frecuencia, amplificando el efecto y haciéndola detectable (Collins 2004).

${ }^{31}$ Sus pares calcularon la máxima fuerza que la radiación gravitacional debía tener a su paso por la Tierra y no coincidian con la sensibilidad del detector de Weber.
} 
De hecho, en la actualidad, el interferómetro es uno de los instrumentos de más alta precisión en la ciencia y ha diversificado ampliamente sus usos. Como resultado indirecto de este desarrollo, varias décadas después los primeros en pensar en el uso de interferómetro como medio para detectar ondas gravitacionales en 1962 fueron los rusos Mikhail Gertsenshtein y Vladislav I. Pustovoit. Sin embargo, es a Rainer Wess del MIT a quien se le atribuye el crédito de su completa conceptualización como dispositivo para ser capaz de detectar ondas gravitacionales, ya que analizó su modo de operación, sensibilidad y posibles fuentes de ruido, además de su primera construcción y desarrollo.

\section{El proceso de detección de ondas gravitacionales por medio de un interferómetro: el proyecto LIGO}

A partir de entonces se comienza consolidar la idea de utilizar el mismo dispositivo que Michelson había inventado para detectar el éter, para observar ondas gravitacionales. Por supuesto, nos encontramos a unos cien años de distancia tecnológica, sofisticación teórica y ante un requisito de sensibilidad mucho mayor. En este caso, una onda gravitacional debe acortar momentáneamente uno de los brazos del interferómetro y alargar el otro y, posteriormente, invertirse el efecto (ver la figura4). En la inversión del efecto las franjas de interferencia del aparato debían moverse hacia atrás y hacia delante a la misma frecuencia a través de la pantalla. El mínimo cambio en la longitud de una de las longitudes del rayo de luz afectaría el patrón de interferencia, de forma similar al caso del éter, pero en esa ocasión debido a la deformación espaciotemporal originada por la onda gravitacional. Si una de las longitudes cambia lo suficiente para que el impacto de una cresta se vuelva valle, la superposición se volverá una cancelación de una franja; las bandas oscuras se volverán brillantes y viceversa, y parecerá que el patrón de interferencia se mueve hacia los lados a través de la pantalla. Sin embargo, como las ondas gravitacionales son tan débiles, la sensibilidad del interferómetro usado en este experimento debía ser un billón de veces mayor que la del utilizado por Michelson y Morley ${ }^{32}$ (Collins 2004, pp. 269-270).
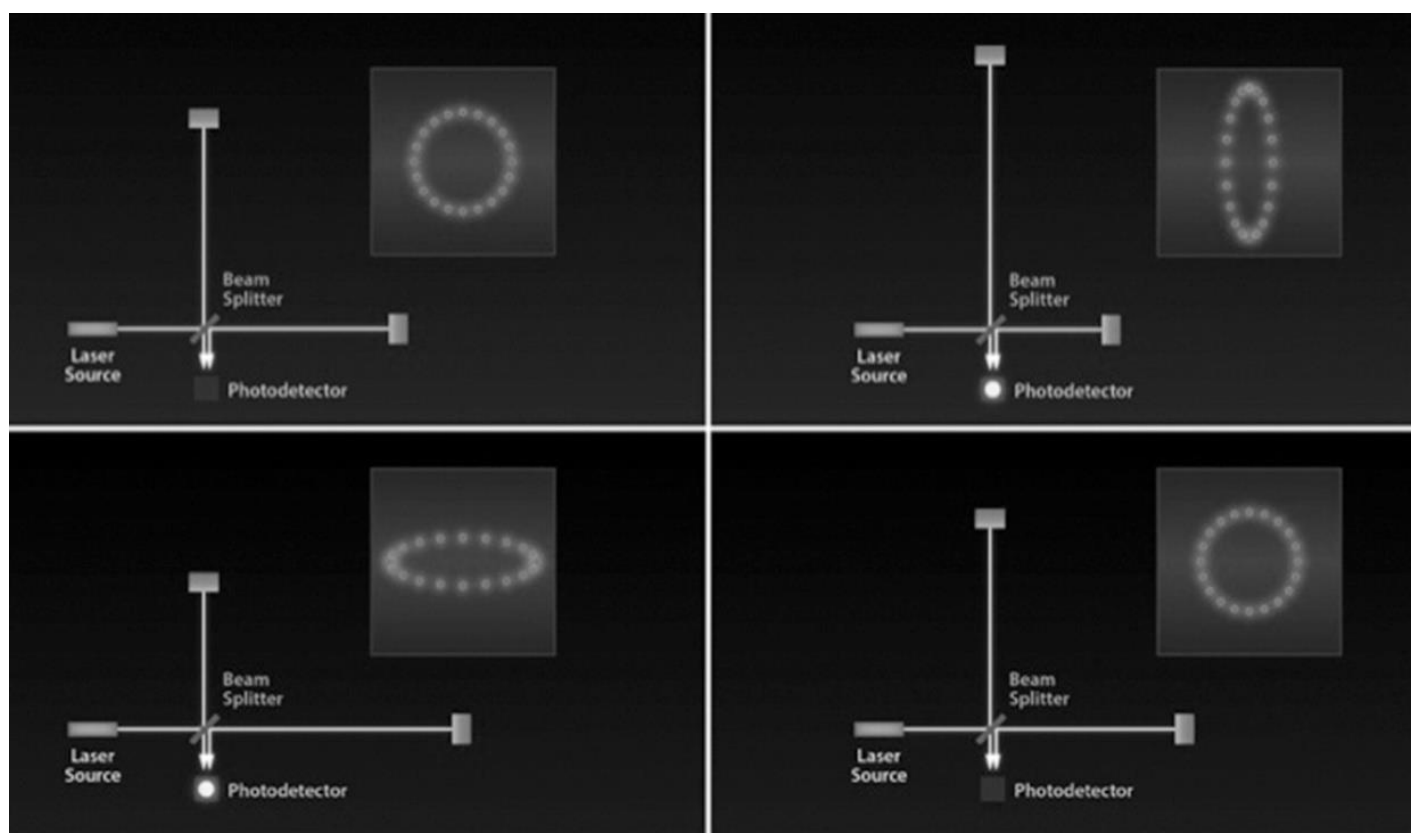

Figura 4. Efecto del paso de una onda gravitacional en la geometría espacial y, como consecuencia, en los brazos de un interferómetro.

\footnotetext{
${ }^{32}$ Las estimaciones de las ondas producidas por los eventos violentos en galaxias cercanas implican que al llegar a la Tierra éstas producirán cambios de $1 / 10^{21}$ que es aproximadamente la millonésima parte del tamaño de un protón. Para ver las cuestiones más técnicas del proyecto LIGO (ver Collins 2014, cap. 1).
} 
Después de las diversas controversias en torno a los hallazgos con la técnica de barras, para principios de la década del 2000 la interferometría se posicionó como dominante en el campo. ${ }^{33}$ Así, el proyecto de LIGO (Laser Interferometer Gravitational Observatory) fundado por la National Science Foundation (NSF), operado por Caltech y MIT, y en la que participan diversas universidades, nace en medio de esta complejidad teórica y técnica, que incluye dimensiones sociológicas y psicológicas, como veremos.

Una vez aprobado el proyecto, las consideraciones tecnológicas para alcanzar la sensibilidad necesaria, como hemos mencionado, eran muchas y muy finas. La sensibilidad del instrumento depende de la longitud de las trayectorias por las que los rayos viajan. Mientras que el dispositivo del siglo XIX tenía una longitud de unos 3 metros que se recombinaban para amplificar el efecto, los dispositivos modernos tienen brazos de unos 4 kilómetros de largo que se reflejan para producir trayectorias de 100 kilómetros (Collins 2014, p.270). Así, el proyecto LIGO tiene dos interferómetros que se encuentran a $3000 \mathrm{~km}$ de distancia, ${ }^{34}$ en Washington y Louisiana respectivamente, con tales brazos y tubos de $1.5 \mathrm{~m}$ de diámetro (la fotografía1 muestra uno de los interferómetros). Estos interferómetros tienen espejos aislados de ruido sísmico, láseres potentes al vacío, cuentan con sofisticados sistemas de amortiguamiento para aislar buena parte de las perturbaciones externas. Asimismo, mientras más brillosos sean los rayos, más fina es la medición ya que se observa mayor contraste entre las zonas oscuras y brillantes y más legibles son los cambios. Por otro lado, la luz deber ser monocromática, pura y estable, para que las franjas sean definidas, afectadas sólo por los cambios en la longitud de la trayectoria y no por cambios en la fuente. Sin embargo, existen numerosas fuentes de ruido en la medición, por lo que el dispositivo requiere de un constante monitoreo y ajuste ${ }^{35}$ que a veces puede ser difícil de separar de la manipulación o manejo a posteriori (Collins 2004, p. 425).

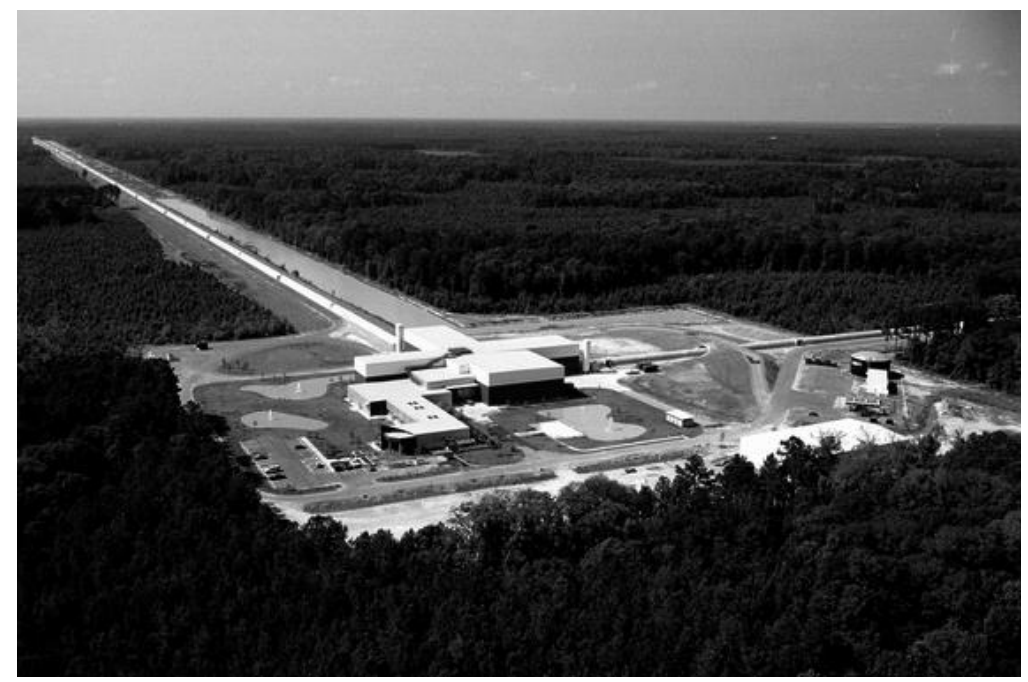

Fotografía 1. Interferómetro de Ligo en Livingston Louisina.

Además, la sofisticación teórica y técnica del fenómeno y lo lejos que éste se encuentra de nuestra experiencia cotidiana, implica que la posibilidad de lo que los físicos denominan su "detección directa" sea, como afirma Collins (2004), la conclusión de una larga cadena de inferencias ligadas a su vez por una sucesión de instrumentos, que requieren no solo la relatividad, sino ciencias auxiliares como la óptica, electrónica, computación, probabilidad, estadística, etc., que exigen complicados supuestos y consensos para leerlos e interpretarlos de tal forma que cada uno de los eslabones deber ser aceptado por la comunidad científica del campo (p. 12). Estos supuestos y consensos dependen y determinan a su vez

\footnotetext{
${ }^{33}$ Para ver la historia de estos controvertidos hallazgos fallidos (ver Collins 2004, p. 350).

${ }^{34}$ De esta forma aseguran que si el fenómeno se detecta en los dos sitios no es debido a una perturbación local y se disminuye notablemente la posibilidad de una coincidencia aleatoria.

${ }^{35}$ Para ver las cuestiones más técnicas del proyecto LIGO (ver Collins 2014, cap.1, y, sobre las fuentes de ruido en la medición, ver Collins 2004, pp. 278-279).
} 
de las diferentes formas de analizar e interpretar los datos, y constituyen lo que Collins en sus estudios sociológicos llama "culturas de evidencia" (evidential cultures), que varían de un grupo científico a otro, o en ocasiones de científico a científico. Dichas diferencias en las culturas de evidencia resultan relevantes en nuestras consideraciones epistemológicas, puesto que estos elementos determinan parcialmente en qué condiciones hay una señal en el interferómetro y se detecta o no una onda gravitacional y, por tanto, aportan piezas clave para analizar la relación entre teoría y experiencia, es decir, bajo qué circunstancias la primera puede ser corroborada por la segunda y cómo depende la segunda de la primera, complementando los elementos que se mostraron en el caso del éter.

Ahora bien, en la detección de las ondas gravitacionales, dado que los científicos tratan de separar una "señal auténtica" del "ruido", cuando la diferencia entre ambos es muy pequeña ${ }^{36}$-como en este caso-, utilizan un procesamiento estadístico que puede ser leído de diferentes formas. Si bien se sabe que mientras más poco frecuente o raro sea el evento es más probable que sea una auténtica "señal", no hay procedimientos aritméticos para decidir qué números representan algo "raro" o algo "frecuente". Por lo tanto, no hay un procedimiento único para decir cuando un número o medición implica una "señal" o "ruido" (Collins 2004, pp. 392-393). De tal forma que, dependiendo de cómo se establezca este criterio, se corre el riesgo de dar un falso positivo o un falso negativo, lo cual es equivalente a la diferencia entre ser conservador o relativamente aventurado en la lectura de los resultados. En este caso, mientras que los grupos de las barras tenían una actitud que optaba por preferir un falso positivo que reportar datos, el grupo de LIGO usaba la actitud inversa. ${ }^{37}$ Dependiendo de estas diferentes culturas que varían en las diferentes disciplinas, subdisciplinas (sociología, física, física de altas energías, etc.) y de grupo en grupo, se pueden establecer diferentes resultados en función del nivel de significatividad estadística que se considere aceptable. Este fue un elemento crucial y de muy difícil desarrollo en el caso de las ondas gravitacionales debido al conjunto de "falsas" detecciones y a la controversia entre técnicas en la lucha por financiamiento, ${ }^{38}$ lo que condicionó la forma de evaluar e interpretar las estadísticas por parte de cada grupo. ${ }^{39}$

Por otro lado, Collins también distingue entre dos tipos de actitudes ante la publicación de evidencia: una individualista y una colectiva. En la primera, es compromiso del autor o el grupo de autores tomar completa responsabilidad de la validez y significado de un resultado científico antes de ser público. En contraste, para los colectivistas la responsabilidad se extiende a la red del dominio con otros individuos y grupos. En ambos casos el árbitro último es la comunidad científica, por supuesto, pero el individualista considera el rechazo o crítica de los resultados de su laboratorio como una falla profesional, mientras que el colectivista piensa que esto es parte del proceso científico (Collins 2004, p. 398). Mientras que el grupo italiano de las barras parecía tener una actitud colectivista, en el grupo americano (LIGO) predomina la primera.

Otra dimensión de la cultura de evidencia es la importancia o relevancia probatoria (evidential significance). Ésta es más alta mientras más grande sea la cadena de inferencias involucradas en la interpretación y más importante sea el hallazgo, pero también implica más mediación teórica y, por tanto, más riesgo de generar oposición o controversia. Es decir, mientras más alta es la relevancia

\footnotetext{
${ }^{36}$ Es decir, se trata de discernir que un minúsculo movimiento del brazo del interferómetro es causado por deformación espacio-temporal o cualquier posible perturbación externa.

${ }^{37}$ Collins sugiere algunas razones de tipo psicológico por las que Weber y el grupo de barras tomaron esta actitud. En primer lugar, se trataba de un ingeniero que no fue formado con los estándares de los físicos ortodoxos y, sobre todo, los criterios de los físicos en altas energías. En segundo lugar, como outsider su mejor apuesta era ser aventurado en sus resultados, no teniendo tanto que perder y pudiendo ganar atención. Finalmente, habiendo participado en la Segunda Guerra Mundial como oficial en un submarino, resulta normal que en una situación de guerra se prefiera elegir un falso positivo a un falso negativo, lo cual en ese contexto resulta fatal (2004, p. 394).

${ }^{38}$ Puesto que la tecnología de la interferometría necesitaba fondos tan altos (para 2015 unos 200 millones de dólares) justificar dicha inversión suponía mostrar su superioridad técnica y ello implicó ser particularmente duros con los resultados de las barras (Collins 2004, pp. 297298).

${ }^{39}$ Otra diferencia concierne a las diversas formas en que la evidencia puede ser incorporada en el análisis estadístico, de acuerdo con las perspectivas bayesiana y frecuentista. De tal forma que ciertos resultados estadístico pueden significar diferentes cosas (Collins 2014 , cap. 5).
} 
probatoria es más alto el riesgo interpretativo. Mientras más baja es menos arriesgada, pero menos importante. El grupo italiano tomaba la segunda actitud, mientras que el grupo LIGO la primera; este último no pensaba en anunciar nada a menos que no estuvieran seguros que se trataba de una onda gravitacional (Collins 2004, p. 402).

Sintetizando estos elementos en el caso que nos ocupa, al grupo LIGO le caracterizó un bajo colectivismo, una alta importancia probatoria y un alto umbral estadístico, es decir, lo que Collins denomina una "cultura de evidencia cerrada", la cual tiende a restringir el acceso a sus datos hasta que han sido altamente procesados. Dado que al grupo italiano le caracterizó la actitud opuesta, las diferencias interpretativas entre ambos grupos y la disputa sobre ambas técnicas resultó fundamental. Estos y otros elementos narrados por Collins $(2004,2011)$ se cristalizan en actitudes, normas, y hábitos que en muchas ocasiones se integran en las instituciones y constituyen una determinada forma de coherencia social en las distintas etapas del análisis de datos (Collins 2004, p. 667). Así, su análisis muestra un conjunto de condiciones y presiones culturales e institucionales que determinaban las diferentes formas en que un dato adquiere significado mediante su procesamiento e interpretación. Todas estas mediaciones teóricas de muy distinta naturaleza tienen impacto en establecimiento de la verdad astrofísica, puesto que de todas ellas depende la afirmación o el rechazo de la detección de ondas gravitacionales, i.e., que el experimento nos confirma la teoría.

Este proceso interpretativo, desde el dispositivo hasta el artículo que publica los resultados, incluye todas las transformaciones que ocurren de las señales que pasan a través del aparato, los cambios, amplificaciones y los signos digitales que son arrojados y a su vez transformados, de diversas formas posibles, en el análisis estadístico. Mientras más débil es el fenómeno que observamos, más transformaciones sufre antes de ser "visto". Por lo que en este caso tenemos una cadena larga y muy delicada porque en cada paso hay supuestos, dudas o disputas que pueden poner en duda la veracidad del resultado final (Collins 2004, p. 7). Tan elaborado es el proceso, que, tal y como afirma Collins, la observación directa -de las ondas gravitacionales, por ejemplo- implica acordar en algún punto, dejar de dudar en esta cadena de inferencias. En definitiva, como él mismo afirma: "ver" -en las ciencias- es acordar que se ve (Collins 2004, p. 7). Así lo muestra la detallada y sofisticada sociología de la detección de las ondas gravitacionales de Collins, en la cual analiza con sumo cuidado la forma en que se aprende a ver a través de estos sofisticados instrumentos, y la forma en la que se forman los consensos y las controversias en torno a los datos obtenidos por diversos grupos que obedecen a culturas científicas que no son homogéneas.

Así, existen numerosas sutilidades teóricas implicadas en el decidir si algo que se detecta son datos que provienen de la detección del fenómeno esperado. Por lo tanto, una detección está condicionada, revestida de supuestos controvertibles y no es una lectura auto-contenida y auto-determinada por las señales detectadas (Collins 2014, p. 20). De tal forma que, en la frontera y alcance de nuestra observación los objetos se vuelven cada vez más tenues y el carácter indirecto de estas observaciones se estira de tal forma que el propio concepto de observación parece llegar a un punto crítico (Collins 2004, p. 93).

La muestra final y más contundente de la larga cadena de mediaciones que derivan en una "observación" de ondas gravitacionales se evidencia en que el entrenamiento realizado por este equipo para la detección de las ondas incluyó un test deliberado de habilidades y disposición ante la detección conocido como blind injection challenge en el que se emitían falsas señales en los interferómetros de forma y magnitud desconocida de acuerdo con una secuencia de números al azar: el trabajo del equipo era encontrarlos y analizarlos, siempre sabiendo que podrían ser completamente falsos ${ }^{40}$ (Collins 2004, p. 21). Es decir, se entrenaban teórica, técnica y psicológicamente a través de simulaciones de mediciones reales.

\footnotetext{
${ }^{40}$ Se trataba de calibrar el proceso completo de la detección, incluyendo la disposición y actitud psicológica del equipo que recibía y analizaba los datos. Por lo que a nuestra consideración podemos leerlo como una especie de condicionamiento ante la difícil distinción entre ruido y señal.
} 
Finalmente, el 11 de febrero de 2015 los científicos del ahora famoso experimento de LIGO anunciaron que la primera observación directa de las ondas gravitacionales se logró el 14 de septiembre de 2015 (Abbott et. al. 2016). Se trataba de la colisión entre dos agujeros negros de 29 y 36 masas solares respectivamente que colisionaron a una velocidad de $.6 \mathrm{c}$, cuya masa final fue de 62 masas solares que se encontraban a una distancia de 1300 años luz (las 3 masas solares de la diferencia son la energía de las ondas gravitacionales). Su frecuencia de oscilación es de unos 100 a $1000 \mathrm{hz}$ por lo que la señal se conecta a una bocina y es posible escuchar la detección (su pico de frecuencia fue a $200 \mathrm{~Hz}$ ) (La figura 5 muestra el registro de la detección.)

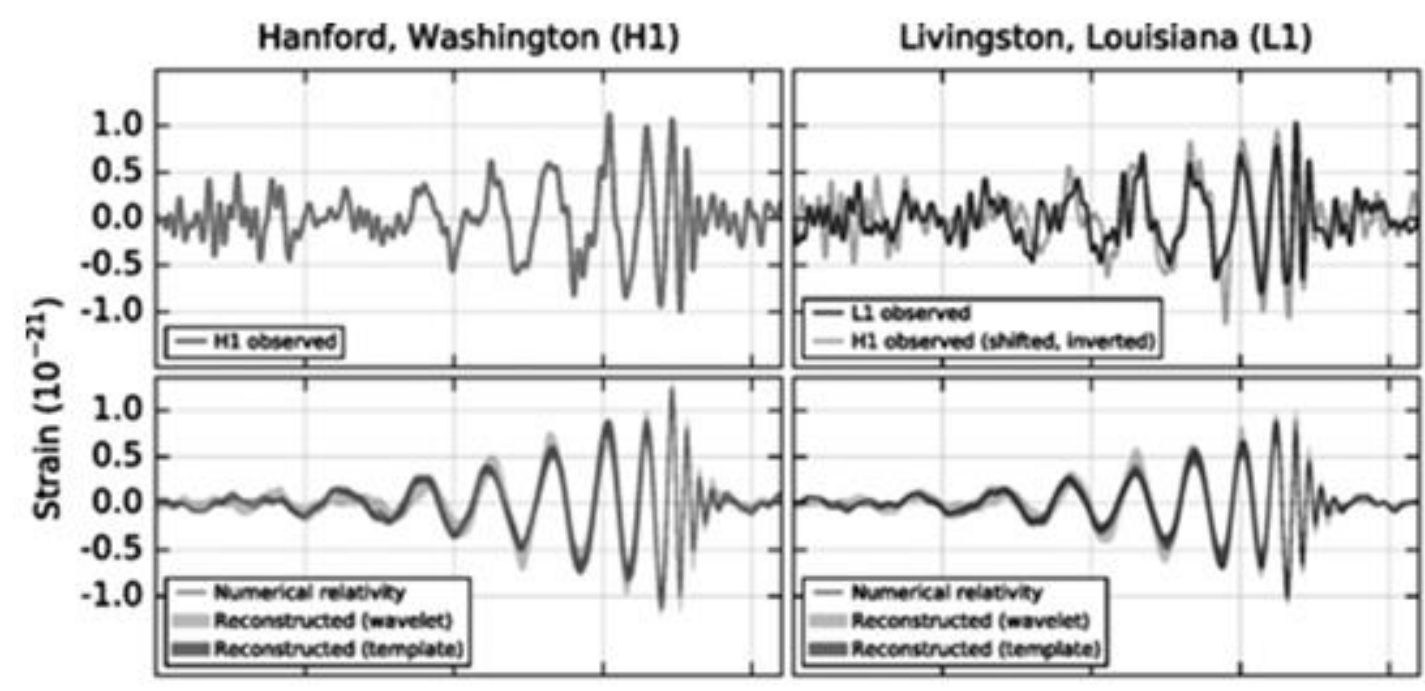

Figura 5. Registro original y "suavizamiento" de las ondas gravitacionales observadas extracto de la Fig. 1 en Abbott (2016).

\section{La relación entre la teoría y la experiencia del espacio en el interferómetro de Michelson}

De una forma mucho más sofisticada y tecnológicamente muy superior, el dispositivo que el observatorio de ondas gravitacionales más grande del mundo utilizó para la medición de ellas fue precisamente un interferómetro de Michelson, compartiendo los mismos principios de funcionamiento que el dispositivo inventando en la década de 1880 para detectar el movimiento de la Tierra respecto al éter. Ambos pretendían leer un patrón de inferencia en el dispositivo, pero que en cada contexto tenía distintos significados. Así, si tenemos como hilo conductor la invención y el uso del interferómetro para indagar sobre las propiedades del espacio en distintos momentos históricos y teóricos -como se ha mostrado-, podemos extraer un breve análisis y conclusiones respecto a la relación entre teoría y experiencia en la ciencia que sustentan determinadas afirmaciones del filósofo austriaco Paul Feyerabend pero -aún másque incorporan elementos sociológicos y psicológicos que no son usualmente considerados en sus análisis.

La característica más evidente que sale a la luz en este caso es la negación de la tesis sobre la autonomía de los hechos, es decir, la idea de que éstos son accesibles independientemente de consideraciones teóricas acerca de ellos -esto es, su disponibilidad al margen de la teoría. La vieja idea del empirismo presupone que existen una clase de hechos que son "dados" de alguna forma. Sin embargo, según Feyerabend hechos y teorías están mucho más conectados de lo que se admite según dicho principio. La idea de la carga teórica -i.e. que todo hecho conlleva una conceptualización indispensable para darle sentido-, está implicada en la conexión de teoría y experiencia. Dicha idea no es exclusiva de Feyerabend, pues incluso los miembros del positivismo lógico sostenían dicha tesis (Ramirez 2006). No obstante, la dependencia entre teoría y experiencia es más radical y tiene mayores 
consecuencias para la concepción del conocimiento en la propuesta de Feyerabend, como veremos a continuación.

En primer lugar, el episodio del experimento de Michelson y Morley nos ha mostrado que su resultado tuvo distintas interpretaciones para dos perspectivas teóricas diferentes: la de Lorentz y la de Einstein. A pesar de ello, ambas pretendían describir los mismos resultados y eran igual de eficientes y racionales para hacerlo. Ello muestra un ejemplo en el que las creencias científicas no se abandonan debido a que se ofrecen razones usando supuestos comúnmente aceptados a favor de alguna. En contraste, se trata de un cambio gradual y no homogéneo en la comunidad científica que conduce a nuevos modelos y formas de ver el mundo -al menos hasta la Relatividad General (Feyerabend 2003, p. $55)-$.

Hemos visto cómo ambas explicaciones se desarrollan en un complicado proceso en el que tienen éxitos y derrotas, y aunque normalmente se supone que los enunciados de observación tienen una función decisiva entre teorías rivales como lo eran estas dos, los resultados de Michelson, i.e. la falta de patrón de interferencia, podía ser justificado por ambas teorías: en una significaba la ausencia de movimiento absoluto y la constancia de la velocidad de la luz, mientras que en la otra significaba la distorsión de las medición espacio-temporales debido al movimiento del marco de referencia. Así, queda fuera de lugar la versión de que este experimento crucial refutó la existencia del éter y dio pie al surgimiento de la relatividad. ${ }^{41}$ En todo caso, fue un elemento que participó en su futuro surgimiento y el resultado solamente sirve como elemento pedagógico y de justificación para ilustrar la inexistencia del éter, una vez admitida la nueva teoría.

Este es un claro ejemplo en el que, como Feyerabend lo señala, no rechazamos una teoría porque contradice los hechos, sino porque contradice una cierta teoría en la cual basamos la construcción de nuestro lenguaje observacional -el clásico o el relativista-. Lo que afirma el austriaco en su texto "Cómo ser un buen empirista”, se cumple en el caso abordado, pues las explicaciones alternativas -en este caso representadas por la propuesta de Einstein- habían de desarrollarse de tal manera que los problemas ya resueltos por Lorentz pudieran ser tratados de nuevas formas, por lo que proporcionarían un parámetro de crítica del esquema asentado (Feyerabend 1999a, p. 80). Pero dicha crítica va más allá de la comparación con los hechos experimentales, pues involucra en definitiva un cambio sobre los principios físicos que dan cuenta de la realidad (simetrías de leyes vs espacio y tiempo absolutos, etc.) y, con ello, nada más y nada menos que a la propia estructura del espacio-tiempo.

En resumen: Einstein introdujo nuevos principios cinemáticos siguiendo determinados supuestos epistémicos y ontológicos que daban cuenta de las dificultades experimentales de forma distinta a la convencional. Con ello su teoría trastocaba los principios clásicos en los cuales la ontología del lenguaje observacional de Lorentz se basaba. De lo cual podemos concluir, junto con Feyerabend, que la lógica de los términos observacionales no se agotaba por los procedimientos que estaban conectando con su aplicación con base en la observación, sino que dependían de las ideas generales que determinaban la ontología de cada teoría. Estas ideas eran disputadas sin que ningún procedimiento observacional se viera afectado (Feyerabend 1999a, p. 87) y su aceptación dependía también de preferencias teóricas de cada individuo, grupo o cultura científica. Las consideraciones teóricas einsteinianas sobre la realidad como la simetría y simplicidad- que le guiaron a configurar su teoría, generaron una actitud empírica distinta de la estándar que, una vez desarrollada y aceptada, se estableció como argumento empírico.

La perspectiva de Feyerabend en este respecto también da cuenta de la lectura a posteriori del resultado experimental, pues una vez aceptada la relatividad éste es interpretado y estandarizado como una "refutación" de la existencia del éter, ya que dicha interpretación está realizada en términos de la teoría vigente, ${ }^{42}$ además de omitir elementos históricos de este cambio científico (Feyerabend 1981a, p. 32).

\footnotetext{
${ }^{41}$ Como explicamos antes, no lo refutó si consideramos que la existencia del éter (p) no necesariamente implicaba un resultado positivo en M\&M (p). Por lo que p no necesariamente implicaba q, y entonces $\neg \mathrm{q}$ no necesariamente niega $\mathrm{p}$.

${ }^{42}$ Dicha interpretación resulta del hecho de que, una vez admitida e instituida la teoría de la relatividad y con ella desechada la existencia del éter, las dudas sobre el carácter concluyente del experimento se disipan, así como la posibilidad de una caracterización alternativa de éste
} 
Asimismo, explica que la impresión o intuición de que cada hecho (en el sentido de datos en sí mismos) sugiere sólo una explicación (la vigente) y el hecho de que nuestras teorías parecen determinadas por los hechos surge debido a que cuando se ha sostenido un punto de vista o teoría durante mucho tiempo, éste se convierte en familiar o no controvertido, e influye en nuestras expectativas acerca del lenguaje y las percepciones (Feyerabend 1981a, p. 34). Esto a su vez explicaría la resistencia de una buena parte de los físicos experimentales y teóricos a abandonar la existencia del éter a finales del siglo XIX -la existencia de una perturbación electromagnética en el vacío resultaba poco familiar o intuitiva-.

Como conclusión parcial, podemos decir que requerimos de supuestos teóricos que van más allá del contenido empírico en la explicación de la experiencia, que además son inseparables de su contenido científico (Feyerabend 1981b, p. 43). Éste último está constituido por todos los elementos que nos proporcionan la capacidad de prueba o contrastación del fenómeno y que, sin embargo, no toma en cuenta distintos elementos del contenido empírico, de la experiencia en general, que pueden influir las observaciones y mediciones, como son las perturbaciones que se ignoran o las simplificaciones que se realizan, si bien parte de la hipótesis de que éstos se conocen y ello posibilita a su vez realizar dichos supuestos.

Asimismo, la co-existencia de teorías inconsistentes, pero empíricamente adecuadas es un elemento esencial en el desarrollo científico pues desarrolla el sentido crítico hacia las descripciones instituidas, como ocurrió en este caso (Feyerabend 1981c, p. 76). Sin embargo, a diferencia de lo que a veces parece promover Feyerabend, esta proliferación y coexistencia de alternativas no es algo que se tenga que mantener a priori de forma permanente, sino siempre en función de los problemas empíricos que enfrentamos, como en los casos abordados.

El ejemplo del interferómetro nos muestra un mismo procedimiento experimental con resultados diferentes que puede ser leído exitosamente desde tres teorías físicas diferentes: la de Lorentz, la relatividad especial y la relatividad general. Así, los procedimientos experimentales exigen que las afirmaciones de una teoría física sean accesibles a la experiencia, lo cual implica a su vez la construcción de un procedimiento bien definido de confirmación (Feyerabend 1999b, p. 18). En particular, el ejemplo de la medición de las ondas gravitacionales muestra que cuando medimos entidades científicas, la transición para llegar a lo que denominamos observación involucra aparatos de medida que exigen una interpretación y acuerdos sobre ésta a muy diferentes niveles, entre ellos el sociológico y psicológico inclusive para definir las características de lo medido-. Sin embargo, como afirma Feyerabend, una vez que se establece y estandariza es cuestión de tiempo hasta que el proceso se vuelve familiar en la comunidad científica, y termina por no haber una distinción consciente entre la presencia del conjunto de criterios y de los hechos. Esto ilustra perfectamente los procedimientos establecidos en la detección de ondas gravitacionales que son determinados por teoría, aparatos, diferentes formas de análisis, administración e interpretación de datos y hasta una cierta predisposición por parte de los astrofísicos. Eventualmente, la presencia de todos estos criterios no se considera más por sí misma, sino que inmediatamente se dice que aparece la onda gravitacional. Entonces se dice que ésta es directamente observable, como ha ocurrido.

Así, la detección de las ondas gravitacionales se convierte en un buen ejemplo de que la indubitabilidad de ciertas afirmaciones y hallazgos es también una cuestión de entrenamiento y de instrucción regular, de la construcción de consensos teóricos (en física, óptica o estadística) pero también sociales -como lo es la cultura de evidencia-, tal como se aprecia en el análisis de Collins. Con ello negaríamos, como Feyerabend, el supuesto de los datos sensoriales como fundamento del conocimiento teórico, pues las circunstancias de observabilidad de las perturbaciones espacio-temporales son el resultado de un entrenamiento teórico, técnico y social en el cual la existencia y la accesibilidad empírica de dichas entidades está ya supuesta. Ello implica que la creencia en la relatividad general y con ella en

al que supone M\&M. Es decir, parte de una mirada retrospectiva que ya da por hecho que no existe el éter, por lo que omitiendo los elementos históricos aquí descritos se vuelve plausible pedagógicamente decir que la medición negativa del interferómetro ( $\neg \mathrm{q})$ "refutaba” la existencia del éter ( $\mathrm{p}$ ) (es decir que $\mathrm{p} \rightarrow \mathrm{q}$ y por lo tanto $\neg \mathrm{q} \rightarrow \neg \mathrm{p}$ ), y ello daba pie al surgimiento de la relatividad. 
las ondas gravitacionales nos lleva a cierta disposición teórica -en un sentido amplio- de los datos sensoriales mismos. Esto no es otra cosa que su interpretación, sin la cual los datos mismos carecen de sentido. En palabras de Feyerabend: "[P]sicológicamente hablando, los datos sensibles son el resultado de nuestra creencia en la existencia de ciertas entidades teóricas"43 (Feyerabend 1999b, p. 29).

De tal forma que la evidencia experimental siempre consiste en hechos analizados, modelados y manufacturados de acuerdo a alguna teoría, ayudada por teorías o premisas auxiliares (Feyerabend 1981c, p. 61). Muestra de ello son, de entrada, las correcciones y selección que aplicamos a las medidas que, como vimos en proceso de detección de las ondas gravitacionales, puede ser diferente según determinados supuestos sobre los dispositivos y las señales que arroja, interpretaciones del tratamiento estadístico y, más aún, en función de los acuerdos implícitos o explícitos en diferentes culturas de evidencia. Así, los hechos que cuentan como evidencia para establecer la confirmación de una teoría y con ella, una verdad científica, pueden ir cambiando de acuerdo a criterios de distinta naturaleza (técnicos, estadísticos, sociales, etc.), como lo refleja el contraste entre el grupo de las barras y el de LIGO.

De forma más general, la historia de estos experimentos nos muestra que las entidades científicas están atadas a la teoría, la cual a su vez está insertada en una determinada cultura, que a su vez los postula y proyecta (Feyerabend 2003, p. 57). Por lo que un sistema experimental está envuelto en una red complicada de factores sociales que incluye elementos teóricos, técnicos, sociológicos, psicológicos, etc., que constituyen su fundamento. Por lo tanto, todos los conceptos empíricos son teoréticos, pues hay una larga y compleja justificación de su significado práctico, aunque ésta sea implícita (Feyerabend 1999b, p. 23). Por ejemplo, después de estos episodios científicos, una noción tan intuitiva y aparentemente directa como la longitud ya no es más un concepto absoluto, sino un conjunto de relaciones que son válidas en un sistema de coordinadas específico. Una experiencia básica como está tenemos que interpretarla a luz de las teorías, en este caso de la geometría del espacio-tiempo, pues no hay experiencia neutra, como afirma Feyerabend. Aunque en la interpretación hay cierta invariabilidad práctica que tienen en común diversas teorías que se han propuesto en la historia de la ciencia, no siempre se cumple ésta ni se teoriza de la misma forma (Feyerabend 1999b, p. 48). Paradójicamente, para la historia de la teoría de la relatividad, resultados aparentemente opuestos, es decir, la ausencia del patrón de interferencia en el siglo XIX y su hallazgo en el XXI, contribuyeron de forma distinta, bajo marcos de interpretación diferentes, a su confirmación.

Por lo tanto, siguiendo a Feyerabend diríamos que un lenguaje observacional está completamente especificado por sus características vinculadas a su interpretación (Feyerabend 1981a, p. 19). Lo que la situación experimental u observacional determina causalmente es la aceptación o rechazo de una oración, de un evento físico -en este caso la ausencia o presencia del patrón de interferencia-. Sin embargo, dichas indicaciones no significan nada al menos que tengamos una teoría que nos enseñe qué situaciones esperar en el mundo y qué garantías tenemos en una situación particular, como lo hizo la relatividad general (y el resto de supuestos) para los miembros de grupo LIGO.

Así, "lo dado" o "la experiencia directa", el patrón de interferencia, aparece bajo ciertas condiciones específicas, y ambos resultados apuntan hacia una reestructuración de los conceptos de espacio y tiempo respecto de las nociones clásicas. Por ello, los fenómenos por sí mismos no pueden determinar las interpretaciones (Feyerabend 1981c, p. 51). En esta historia del interferómetro, desde el éter hasta las ondas gravitacionales, hemos cambiado de ontología, por lo que hemos tenido que revisar la interpretación de todas las medidas, el orden de magnitud en el que aparece el fenómeno y lo que éste significa, y con ello la expectativa es que poco a poco se hará autoevidente que éste dispositivo mide ondulaciones espacio-temporales. Pero también posiblemente el contenido y la interpretación de esas mismas mediciones vaya transformándose conforme se desarrolla el campo.

Como afirma Feyerabend,

\footnotetext{
43 “[P]sychologically speaking, sense-data are the result of our belief in the existence of certain theoretical entities" (traducción propia).
} 
el material que un científico tiene realmente a su disposición, sus leyes, sus resultados experimentales, sus técnicas matemáticas, sus prejuicios epistemológicos, su actitud hacia las consecuencias observadas de las teorías que él acepta, este material es indeterminado y ambiguo de muchas maneras, y nunca está completamente separado de la base histórica (Feyerabend 1975 p. 49).

En definitiva, la experiencia se muestra en este ejemplo histórico como algo que surge con contenido positivo junto con las suposiciones teóricas de ésta -no antes-. Sin ellas no tenemos guía para llevar a cabo una acción experimental. No hay observación sin los supuestos teóricos pues ambas están en igualdad (Feyerabend 1981d, p. 134).

Por lo tanto, cualquier teoría está conectada con una cultura y un junto de situaciones históricamente condicionadas, en sus técnicas y supuestos, en tanto la ciencia es parte de ésta. En particular, este ejemplo muestra como el conocimiento alrededor de un dispositivo experimental está históricamente o contextualmente condicionado. Hemos visto también cómo las teorías van más allá del conjunto de datos disponibles en determinado momento y su confirmación empírica siempre implica un margen de error. Ello da cierto margen de liberad o variedad a la construcción de teorías por parte de la propia experiencia, pero dicha libertad y variedad se encuentran atadas también a determinadas prácticas culturales, tradiciones, creencias y prejuicios en la ciencia que pueden estar conectadas con idiosincrasias personales. Todo esto impone condiciones a la significación de la experiencia científica.

Esta relación entre teoría y experiencia, nos permite afirmar, junto con Feyerabend, que el mundo al que accedemos es dinámico, plural, históricamente condicionado, ejerce influencia y a su vez "refleja la actividad de los exploradores" (Feyerabend 2003, p. 64). Pues cada explicación es una interpretación que ordena y adapta resultados empíricos y matemáticos a una perspectiva que se corresponde con una concepción particular de la realidad. Y la realidad entonces, es aquello, dice el austriaco, que desempeña un papel importante en nuestra investigación (Feyerabend 2003, p. 86). Por lo tanto: "Los científicos son escultores de realidad, pero sólo actúan causalmente en sistemas físicos y crean condiciones semánticas generando inferencias sólidas desde efectos conocidos hacia proyecciones nuevas y a la inversa, desde las proyecciones hacia efectos comprobables" (2003, p. 62). Este proceso asentado en prácticas culturales contingentes está siempre inacabado, de tal forma que, como suele decirse, podemos afirmar que la investigación y la conversación nunca terminan.

Bibliografía

Abbott, B. P. et al. (2016), "Observation of Gravitational Waves from a Binary Black Hole Merger", Physical Review Letters 116: 061102.

Bridgman, P. W. (1936), The Nature of Physical Theory, Princeton: Princeton University Press.

Collins, H. (2004), Gravity's Shadow. The Search for Gravitational Waves, Chicago: University of Chicago Press.

Collins, H. (2014), Gravity's Ghost and Big Dog. Scientific Discovery and Social Analysis in the Twenty-First Century, Chicago: University of Chicago Press.

Eddington, A. (1939), The Philosophy of Physical Science, New York: The Macmillan Company.

Einstein, A. (1923), "On the Electrodynamic of Moving Bodies”, en Einstein, A., Lorentz, H. A., Weyl, H. y H. Minkowski, The Principle of Relativity, New York: Dover, pp. 35-65.

Einstein A. y N. Rosen (1937), “On Gravitational Waves”, Journal of the Franklin Institute 223(1): 43-54.

Eisenstaedt, J. (2015), Antes de Einstein: relatividad, luz y gravitación, México: Fondo de Cultura Económica.

Feyerabend, P. K. (1975), Tratado contra el método. Esquema de una teoría anarquista del conocimiento, Madrid: Tecnos.

Feyerabend, P. K. (1981a), “An Attempt at a Realistic Interpretation of Experience”, en Feyerabend, P. K., Realism, Rationalism, and Scientific Method. Philosophical Papers Vol. 1, Cambridge: Cambridge University Press, pp. 17-36. 
Feyerabend, P. K. (1981b), “On The Interpretation of Scientific Theories”, en Feyerabend, P. K., Realism, Rationalism, and Scientific Method. Philosophical Papers Vol. 1, Cambridge: Cambridge University Press, pp. 37-43.

Feyerabend, P. K. (1981c), "Explanation, Reduction and Empiricism”, en Feyerabend, P. K., Realism, Rationalism, and Scientific Method. Philosophical Papers Vol. 1, Cambridge: Cambridge University Press, pp. 44-96.

Feyerabend, P. K. (1981d), "Science Without Experience", en Feyerabend, P. K., Realism, Rationalism, and Scientific Method. Philosophical Papers Vol. 1, Cambridge: Cambridge University Press, pp.132-136.

Feyerabend, P. K. (1999a), "How to Be a Good Empiricist: A Plea for Tolerance in Matters Epistemological", en Feyerabend, P. K., Knowledge, Science and Relativism. Philosophical Papers Vol. 3, Cambridge: Cambridge University Press, pp. 78-103.

Feyerabend, P. K. (1999b), “The Problem of the Existence of Theoretical Entities”, en Feyerabend, P. K., Knowledge, Science and Relativism. Philosophical Papers Vol. 3, Cambridge: Cambridge University Press, pp. 16-49.

Feyerabend, P. K. (2003), Provocaciones filosóficas, Madrid: Biblioteca Nueva.

Holton, G. (1969), "Einstein, Michelson and the 'Crucial' Experiment”, Isis 60: 133-169.

Janssen, M. (2002), "Reconsidering a Scientific Revolution: The Case of Einstein versus Lorentz", Physics in Perspective 4: $421-446$.

Kompaneyets, A. S. (1962), Theoretical Physics, New York: Dover.

Kostro, L. (2000), Einstein and the Ether, Montreal: Apeiron.

Michelson, A. A. (1881), "The Relative Motion of the Earth and of the Luminiferous Ether", American Journal of Science, Series 3, 22: 120-129.

Miller, A. I. (1984), Albert Einstein's Special Theory of Relativity. Emergence (1905) and Early Interpretation (1911), New York: Springer.

Ramírez, S. (2006), "Feyerabend: la tradición empirista y la carga teórica de la observación”, en López, C. (ed.), Perspectivas y horizontes de la filosofía de la ciencia a la vuelta del tercer milenio, México: UNAM, pp. 19-39.

Reichenbach, H. (1958), The Philosophy of Space and Time, New York: Dover.

Staley, R. (2008), Einstein's Generation. The Origins of the Relativity Generation, Chicago: University of Chicago Press.

Swenson, L. S. (1970), "The Michelson-Morley-Miller Experiments before and after 1905", Journal for the History of Astronomy 1: 56-78.

Swenson, L. S (1972), The Ethereal Aether. A History of Michelson-Morley-Miller Aether-Drift Experiments, 1880-1930, Austin: University of Texas Press.

Taylor, J. H. y J. M. Weisberg (1982), "A New Test of General Relativity: Gravitational Radiation and the Binary Pulsar PSR 1913+16”, Astrophysical Journal 1(253): 908-920.

Zahar, E. (1973a), "Why Einstein's Programme Supersede Lorentz's? (I)”, British Journal of Philosophy of Science 24(2): 95. 123.

Zahar, E. (1973b), "Why Einstein's Programme Supersede Lorentz's? (II)", British Journal of Philosophy of Science 24(3): 223-262. 